\title{
Numerical Modeling for In Vivo Electroporation
}

\section{Dejan Šemrov and Damijan Miklavčič}

\section{Introduction}

Electropermeabilization of cell plasma membrane is a threshold phenomena. When a cell is exposed to electric field a spatially dependend transmembrane potential is induced (1). Above a certain threshold value of transmembrane potential permeability of plasma membrane drastically increases. Thus, in order to obtain plasma membrane permeabilization an above threshold transmembrane potential needs to be obtained. This is achieved by an above threshold electric field intensity. Electropermeabilization is therfore characterized by electric field intensity, but also by the duration and number of applied pulses, as well as their shape (2). Electric field intensity of the pulses of selected duration must reach a threshold, typical for a particular type of cell (3). This threshold is also different for the cells in tissue compared to the threshold for electropermeabilization of the membrane of isolated cells (4). Selected electric field intensity, appropriate for electroporation, should at the same time not exceed the value which will cause irreversible permeabilization or even death of the cell (5). This is particularly important for electro-gene transfection.

For the in vitro electroporation of cultured cells in suspension (6) it can be considered, that the cells are exposed to a homogenous electric field, meaning that its direction is uniform and the magnitude of electric field intensity is unique throughout all of the conducting volume. Namely, the distances between the cells in the suspension are very large compared to the diameter of each particular cell (7). Also, the dimensions of the typical chamber, used for the in vitro experiments, and the dimensions of electrodes by which the electric pulses are delivered to the suspension are much larger compared to diameters of the cells and their mutual distance. The electric field intensity can then be 
calculated using a simple formula $E=U / l$, which is the electric field intensity inside ideal capacitor, constructed of two charged parallel plates of infinite surface, where $l$ is the distance and $U$ is the voltage between the plates. For the ex vivo electroporation of tissues or in vivo electroporation in the living organisms, the electric field resulting from the pulse delivery with electrodes usually used (8) can no longer be considered homogenous.

In the case of ex vivo electroporation there is usually only one type of tissue involved, but the pulses are delivered using either plate electrodes of various shapes, placed on the surface of the tissue, or needle electrodes, inserted into the tissue. Both types of electrodes will result in an inhomogeneous field. Furthermore, the biological tissue can not be considered a homogenous conductor. It is composed of cells, which have plasma membranes with very low electric conductivities compared to the conductivity of cytoplasm and extracellular medium. The shape of the cells and their organizations in the tissue are different for each particular type of the tissue (9) thus resulting in different distributions of electric field inside the tissue. In the case of electroporation, the electric conductivity of plasma membrane is increased, which substantially changes the electric phenomena inside the tissue and makes electric field distribution even more complex.

In the case of in vivo electroporation, there are usually many different tissues/organs with different electrical properties involved. Together with the diversity of their geometry, dimensions, and structure this results in even higher degree of inhomogeneity of the electric field resulting from the pulse delivery. The variety of the electrodes' shapes, dimensions and positions used for the in vivo electroporation and the difference in the resulting biological responses $(10,11)$ further point to the difference in electric field distribution resulting from specific electroporation regime.

Mathematical modeling is a new approach in evaluation of the electrical phenomena during electroporation in vivo. Calculation of electric field distribution is a relatively simple, yet efficient tool for the analysis and explanation of experimental results. The influence of electric parameters (shape, dimensions and position of the electrodes), as well as tissue/organ characteristics on the distribution of electric field can easily be evaluated. Different electroporation regimes and experimental conditions can be analyzed and new experiments planned at lower costs. However, all mathematical models must be rigorously validated with adequate measurements of electrical parameters in vivo. Furthermore, mathematical modeling can not replace experimental work, it is merely a source for additional information in the explanation of the phenomena and experimental planning. Anyway, it can contribute to the knowledge and the understanding of the processes involved in electroporation.

Mathematical modeling has a rather long history in the field of biomedical 
engineering and bioelectromagnetism. First reports were published in the sixties (12) and were related to electrocardiology. Electromagnetic field calculation has preserved its importance in the cardiology until now $(13,14)$. The majority of reports dealing with the use of different analytical and numerical calculations were from this field of medicine. In the so called forward or direct problem, the aim of the research was to determine body surface potentials from the known active sources in the heart. The objective of the so called inverse problem was to estimate the source current density in the heart which results in electromagnetic signals measured outside or on the surface of the body i.e. electrocardiograms (ECGs) and magnetocardiograms (MCGs). Calculations of electric fields were also used in the studies of external cardiac pacing (15) and defibrillation (16), where they proved to be an efficient tool for the optimization of electrode systems. Similar approach can be found in the studies of the electromagnetic fields in the human brain $(\mathbf{1 7}, \mathbf{1 8})$, where forward and inverse problems are defined in a similar way relating cerebral electromagnetic sources with the electromagnetic signals outside or on the scalp, that is, magnetoencephalograms (MEGs) and electroencephalograms (EEGs), respectively. Magnetic and electric neurostimulation of the brain were also analyzed using mathematical modeling $(\mathbf{1 9}, 20)$. In oncology, mathematical modeling was widely used in the analysis of the hyperthermia where both the electromagnetic and thermal phenomena were analyzed (21). Electrode configuration (position, sizes and driving voltages) for radio frequency (RF) hyperthermia was also optimized by means of mathematical modeling (22). In the last decade with the increased awareness of the possible health risk effects of electromagnetic fields (cellular phones, power transmission lines, radars, magnetic resonance imaging systems, home appliances, etc.) a lot of research groups were calculating energy absorbed in human body. Many different numerical methods were applied in those studies $(23,24)$ due to diversity of problems in this area of research.

\section{Definitions and Basic Theory}

\subsection{Volume Conductor Theory}

The electric field in the biological tissue, resulting from an application of constant direct electric current, can be considered quasi-stationary, i.e. its time variations can be neglected (25). Its distribution is described by equations for the steady electric currents in the volume conductor. The relation of electric current to voltage and resistance is described by Ohm's law. For the biological tissue, where both positive and negative charge carriers are present with different characteristics, the electric current density $\mathbf{J}$ (units: $\mathrm{A} / \mathrm{m}^{2}$ ) is defined by the point form of Ohm's law: 


$$
\mathbf{J}=\gamma \cdot \mathbf{E},
$$

where $\mathbf{E}$ (units: V/m) is electric field intensity and is defined as the negative gradient of the scalar electric potential $u$ (units: V):

$$
\mathbf{E}=-\nabla u .
$$

$\gamma$ is the electric conductivity of the material (units: $\mathrm{S} / \mathrm{m}$ ) and is in the most generalized case of the anisotropic volume conductor described as a tensor:

$$
\gamma=\left[\begin{array}{lll}
\gamma_{\mathrm{xx}} & \gamma_{\mathrm{xy}} & \gamma_{\mathrm{xz}} \\
\gamma_{\mathrm{yx}} & \gamma_{\mathrm{yy}} & \gamma_{\mathrm{yz}} \\
\gamma_{\mathrm{zx}} & \gamma_{\mathrm{zy}} & \gamma_{\mathrm{zz}}
\end{array}\right] .
$$

When the conductivity of the material can be described in an orthogonal coordinate system $(x-y-z)$ and referred to the principal axes, and both the electric field and the current density are related to the same coordinate system, then all nondiagonal elements are equal to zero and the above matrix becomes diagonal:

$$
\gamma=\left[\begin{array}{lll}
\gamma_{\mathrm{xx}} & 0 & 0 \\
0 & \gamma_{\mathrm{yy}} & 0 \\
0 & 0 & \gamma_{\mathrm{zz}}
\end{array}\right] .
$$

There are two different conductivities in the anisotropic biological tissue (skeletal and cardiac muscles), the parallel or longitudinal, and the perpendicular or transversal. The directions are referred to the orientation of the muscle fibers. If the muscle tissue is oriented along one of the principal axes in the coordinate system, two of the elements in the above matrix, describing conductivities in the perpendicular direction, are equal. Computation of the electric field is simplified if the model can be described in cylindrical co-ordinate system.

Boundary conditions across interface of two volume conductors with different conductivities are:

$$
J_{\mathrm{n} 1}=\mathrm{J}_{\mathrm{n} 2},
$$

where $J_{\mathrm{n} 1}$ and $\mathbf{J}_{\mathrm{n} 2}$ are the normal components of $\mathbf{J}$ at the boundary surface in the volume conductors 1 and 2, and:

$$
E_{\mathrm{t} 1}=E_{\mathrm{t} 2},
$$

where $E_{\mathrm{t} 1}$ and $E_{\mathrm{t} 2}$ are the tangential components of $\mathbf{E}$ at the interface.

The equation of the continuity for current states that free charge cannot remain within a conductor and is the field equivalent of Kirchoff's current law, which states that the net current leaving a junction of several conductors is zero. The continuity equation in the differential form is:

$$
\nabla \cdot \mathbf{J}=0 .
$$


Considering the point form of Ohm's law Eq. 1 we obtain:

$$
\nabla \cdot(\gamma \cdot \mathbf{E})=0 .
$$

Using the definition of the electric field intensity Eq. 2 yields:

$$
\nabla \cdot[\gamma \cdot(-\nabla u)]=0
$$

If the volume conductor is homogeneous and isotropic ( $\gamma$ is scalar), then the above equation becomes Laplace's equation:

$$
\nabla^{2} u=0 .
$$

Equation $\mathbf{1 0}$ is a partial differential equation of elliptic type which together with two types of boundary conditions describes electric field inside the volume conductor. The two types of boundary conditions are: the Dirichlet boundary condition defined as a fixed scalar electric potential, that is, applied voltage on the surface of the model:

$$
u=\bar{u},
$$

and the Neumann boundary condition defined as a first derivative of the scalar electric potential in the direction normal to the boundary surface of the model,that is, current density flowing in/out of the model in the direction normal to the surface, divided by the conductivity of the tissue:

$$
q=\frac{\partial u}{\partial n}=-\frac{\overrightarrow{\mathbf{J}}_{\mathrm{n}}}{\gamma} .
$$

Laplace's equation can be solved analytically or using one of the numerical methods. The analytic method is suitable when the geometry, inhomogeneities and anisotropies of the volume conductor are describable in the same coordinate system, i.e. Cartesian, spherical or cylindrical. Models in which this condition is not fulfilled result in complicated systems of equations which are difficult to solve. Majority of numerical methods, on the other hand, allow approximation of any geometry, material properties, and boundary conditions. Most methods also allow definition of various material inhomogeneities and in some methods anisotropies can be defined. Complex geometry, inhomogeneities and anisotropies of the tissue are characteristic properties of most of the biological systems. The use of numerical techniques is therefore more appropriate in such studies.

\subsection{Finite Element Method}

The finite element method has proven to be very effective in numerous computations of the electric field inside biological systems $(\mathbf{2 6 , 2 7 )}$. The discretization of the domain is the first step in majority of the numerical 
methods. In the so called domain methods, for example, finite element or finite difference, the whole volume of a three dimensional model has to be discretized. In the so called boundary methods, e.g. boundary element method, only boundary surfaces bounding regions of the model with the same material properties, for example, specific biological tissue (skin, muscle, etc.), have to be discretized. The discretization of the domain in finite element method involves the decision about the type, number, size, and shape of the finite elements to model the real biological system, for example, tissue, organ, whole or part of the body. There are no theoretical guidelines on how to perform this step. It is merely an art which depends on the use of engineering judgment, but can produce inaccurate results if not done properly.

The next step of the finite element method approach is the selection of the electric quantity which will be calculated and from which all other electrical quantities will be derived. The scalar electric potential $u$ is a good choice in the volume conductor calculation since it results in less complex calculations. The continuous function of electric potential $u$ is then approximated by a discrete model composed of a set of piecewise continuous functions, usually polynomials, defined over each particular finite element. Appropriate material properties, i.e. electric conductivities, have to be assigned to each particular element. Anisotropic conductivities can as well be applied and result in a specific type of finite element i.e. different function. This is a very important step since it has a strong influence on the results. Several reports about electrical properties of living tissues can be found in the literature $(28,29)$. Then the boundary conditions are applied. As described in the previous subsection, two types of boundary conditions apply in the volume conductor modeling: fixed scalar electric potential and/or first derivative of the scalar electric potential in the direction normal to the boundary surface of the model, that is, current density flowing in/out of the model in the direction normal to the surface, divided by the conductivity of the tissue. In the modeling of in vivo electroporation, different positions and electrode shapes in models can be represented by applying appropriate boundary conditions, for example, correspondent values of electric scalar potential.

After this a set of simple equations describing the discretized scalar electric potential throughout the whole volume of the model is obtained. After rearranging, this system of equations is solved using one of the appropriate numerical methods. At the end, all other desired electrical quantities are calculated from the resulting discrete values of scalar electric potential. The finite element method is described in greater detail in numerous references $(\mathbf{3 0 , 3 1 )}$. 


\section{Analysis of Electric Field Distribution in Electrochemotherapy}

As it was mentioned in the introduction, various electrode geometries were used in the in vivo studies involving plasma membrane electropermeabilization. In electrochemotherapy, membrane permeabilization is used to enhance delivery of chemotherapeutic drugs into the tumor cells and increase antitumor effectiveness. Namely, some chemotherapeutic drugs used in cancer therapy have poor access into the tumor cells. Furthermore, lower systemic doses of chemoterapeutic drugs are required for successful therapy and thus adverse side effects are reduced. Numerical modeling can be used as a tool for the explanation of the observed effects.

In one of the several studies of the efficacy of electrochemotherapy of solid tumors, performed in Ljubljana (10), it was demonstrated that changing electrode orientation improves the efficacy of electrochemotherapy of solid tumors in mice (see also Chapter 14 by Cemazar). Ehrlich ascites tumor in CBA mice was used as a tumor model. When the tumors reached approximately $40 \mathrm{~mm}^{3}$ in volume, they were treated with the combination of intravenously injected Bleomycin (Mack, Germany) and trains of square-wave high voltage DC pulses (amplitude $1040 \mathrm{~V}$, pulse width $100 \mu$ s, repetition frequency $1 \mathrm{~Hz}$ ). Electric pulses were delivered by two parallel stainless steel plate electrodes $8 \mathrm{~mm}$ apart (two stainless steel strips, $7 \mathrm{~mm}$ in width with rounded tips). Good contact between the electrodes and the skin was ensured by means of conductive gel. Tumors were treated either with 4,8 , or $4+4$ pulses in train. In the last group the second train of 4 pulses was delivered with electrodes oriented perpendicularly with respect to the first one with a time interval of $1 \mathrm{~s}$ between the two trains of pulses, that is, $4+4$ pulses. This changing of the electrode orientation resulted in improved anti-tumor efficacy of the electrochemotherapy: prolonged tumor growth delay and higher percentage of short and long term complete responses of the tumors.

\subsection{Finite Element Model of a Mouse With Subcutaneous Solid Tumor}

In order to explain improved antitumor effectiveness in changing electrode orientation experiments and to gain more detailed insight into electrical phenomena inside tumour and in the surrounding tissue during different electrochemotherapy regimes, a three-dimensional (3D) anatomically based finite element model of the mouse with injected subcutaneous solid tumor was built (32). The geometry of the model was based on the 14 cross section scans of a typical animal with a subcutaneous tumor obtained by magnetic resonance 


\begin{tabular}{|c|c|}
\hline Tissue & Conductivity $\gamma(\mathrm{S} / \mathrm{m})$ \\
\hline Skin & 0.04 \\
\hline Fat & 0.046 \\
\hline Muscle & $\begin{array}{l}\gamma_{\mathrm{xx}}=0.225 \\
\gamma_{\mathrm{yy}}=0.225 \\
\gamma_{\mathrm{zz}}=0.9\end{array}$ \\
\hline Bone & 0.025 \\
\hline Connective tissue & 0.025 \\
\hline Intestine & 0.55 \\
\hline Kidneys & 1.01 \\
\hline Liver & 0.333 \\
\hline Lungs & 0.07 \\
\hline Heart & $\begin{array}{l}\gamma_{\mathrm{xx}}=0.2 \\
\gamma_{\mathrm{yy}}=0.2 \\
\gamma_{\mathrm{zz}}=0.9\end{array}$ \\
\hline Tumor & 0.125 \\
\hline
\end{tabular}

imaging (MRI). The distance between the two neighboring cross sections in the longitudinal direction was $2.7 \mathrm{~mm}$. There were eleven MRI scans in the abdominal and three in the thoracal part of the body of the mouse. Resulting three-dimensional geometric structure was built of eleven different tissues (organs), that is, skin, fat, skeletal and heart muscles, bone, connective tissue, intestine, kidney, liver, lung and tumor. Each of these tissues (organs) formed a closed region and a mesh of 3D finite elements was generated inside with appropriate material properties assigned to the elements in each region. Anisotropic characteristics were considered for skeletal and heart muscles, while all other tissues (organs) were modeled as isotropic. The values of the electric conductivities of tissues (organs) used in the model were collected from literature and used in one of previous studies where similar model was verified with the measurements of electric potential in the 5 points in the tumor and surrounding tissue (27). All tissue conductivities are listed in Table 1. Resulting 3D model was made of 7089 3D finite elements which were defined by 7578 grid points. The cross section through the tumor in which the results were observed is shown in Fig. 1.

Different electrode orientations were obtained by applying appropriate boundary conditions in the grid points on the outer surface of the model corresponding to each of the two electrodes. Increased area with the same electric 


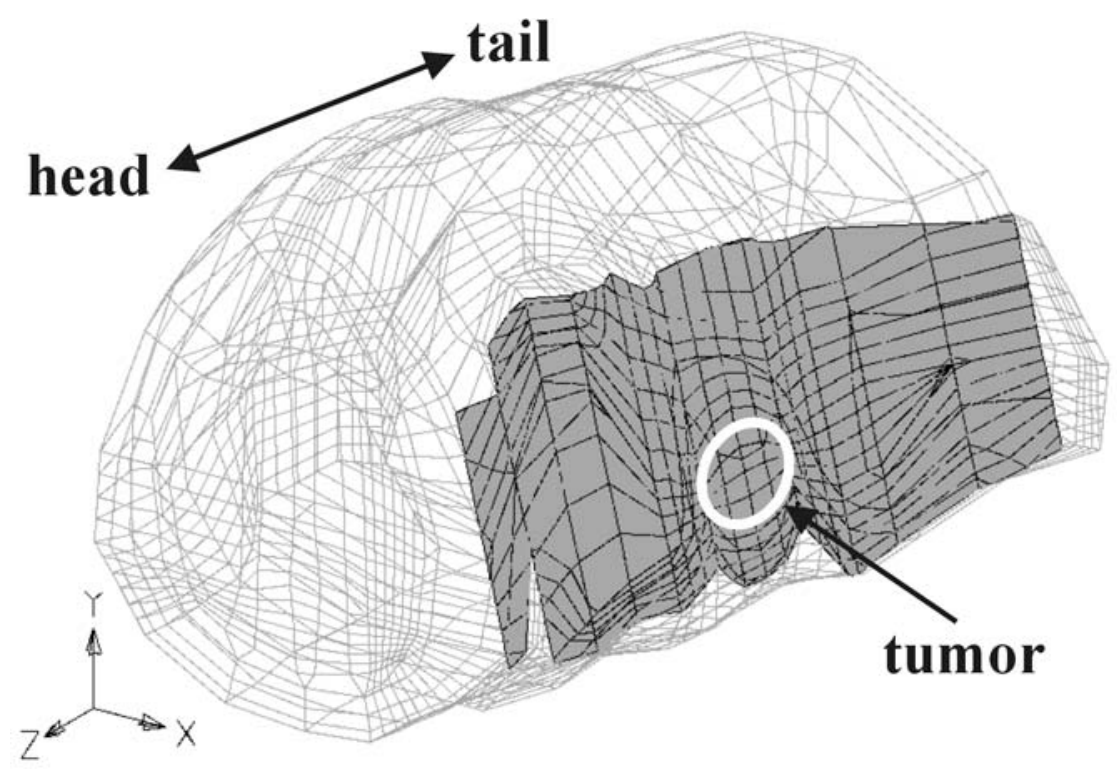

Fig. 1. Three-dimensional anatomically based finite element model of a mouse with subcutaneous solid tumor. Shaded plane represents cross section through the middle of the tumor in which the results were shown.

potential under each electrode resulting from the use of conductive gel was also considered. Fixed values of scalar electric potential, that is, Dirichlet boundary conditions, were assigned to grid points in the regions where electrodes were placed. Two electrode configurations were modeled according to the position of the electrodes with respect to the tumor, e.g. cranial/caudal and dorsal/ventral. Potentials of $0 \mathrm{~V}$ and $1040 \mathrm{~V}$ were assigned to groups of appropriate grid points of the finite element mesh corresponding to each of the electrodes thus modeling the conditions in the experimental study. The width of both electrodes was $7 \mathrm{~mm}$ and their thickness was $0.9 \mathrm{~mm}$. In the cranial/caudal electrode configuration the positive electrode was placed on the left side of the tumor in the direction towards the head of the mouse. The distance between the electrode and the edge of the tumor was $0.9 \mathrm{~mm}$. The negative electrode was placed on the other (right) side of the tumor in the direction towards the tail of the mouse and the distance between the electrode and the edge of the tumor was again $0.9 \mathrm{~mm}$. In the dorsal/ventral electrode configuration the positive electrode was placed above the tumor in the direction towards the back of the mouse. The distance between the electrode and the edge of the tumor was $0.9 \mathrm{~mm}$. The negative electrode was placed on the other side below the tumor 
and the distance between the electrode and the edge of the tumor was again $0.9 \mathrm{~mm}$. On the remaining outer surfaces of the model, the Neumann boundary condition was applied. This boundary was considered as the interface between a conducting medium and air (assumed as an ideal dielectric). Since the conductor (skin layer) was linear and isotropic, the usual Neumann condition was applied i.e. the normal derivative of the electric potential on the interface between the model and surrounding air was zero.

\subsection{Validation of the Model}

The process of the mathematical model validation is of utmost importance. A very basic method of validating in vivo models is simply to compare measured and calculated values of total currents flowing in/out of the model and correspondent potentials/voltages for each particular experimental regime. If there is high correlation between calculated and measured values, the model can be considered a good approximation of the actual electrical phenomena inside biological tissue.

In order to validate previously described model in greater detail current density distributions in tumor were qualitatively evaluated for different specific electrode sets by means of magnetic resonance current density imaging (33). Electric current density imaging (CDI) is a magnetic resonance imaging (MRI) technique that images current density by acquiring data from phase shifts in proton precession. Phase shifts are caused by short pulses of direct electric current, which are passing through the sample during imaging time $(34,35)$. In this study, four point copper electrodes with diameter of $0.6 \mathrm{~mm}$ were used, two by two placed on each cross-plane of the tumor. The point electrodes were connected to the voltage amplifier as shown in Fig. 2. Two configurations of electrodes were thus used: the $2+2$ electrode set, where all four electrodes were connected to the voltage amplifier (Fig. 2c); and the $2+1$ electrode set, where just three electrodes were connected to the voltage amplifier as depicted in Fig. 2b. These two electrode configurations where chosen in order to produce notably different current density and thus electric field distribution in the observed plane (dashed line in Fig. 2) of the tumor as well as in the whole tumor. This difference allowed us to validate the model by means of current density imaging (CDI) and electrochemotherapy effectiveness. CDI was used to map spatial distribution of electric currents through mice tumors. Mice were placed into a stereotactic frame and four point electrodes in pairs two by two with a small amount of conductive gel were affixed to the tumor. The electrodes were connected to a DC voltage amplifier and the voltage applied to the tumor was $50 \mathrm{~V}$ with total duration of current pulses $10 \mathrm{~ms}$. Throughout the imaging time electric current was controlled with an oscilloscope and a voltmeter. To construct a map of electric current density spatial distribution $\left(j_{z}\right.$ in 
b)

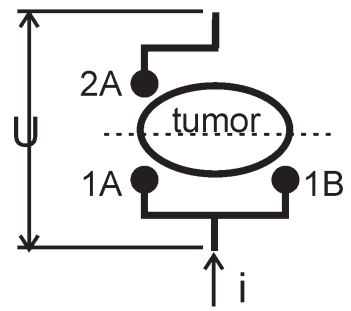

$2+1$ electrode set

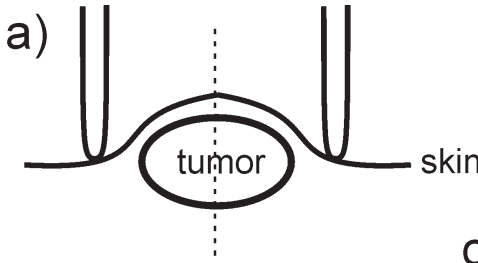

c)

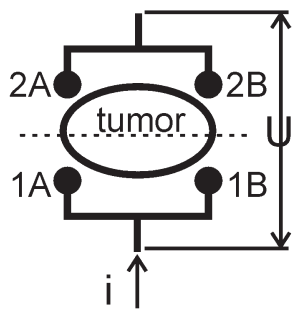

$2+2$ electrode set

Fig. 2. Schematic presentation of $2+1$ and $2+2$ electrode set positioning with respect to the tumor and point electrodes electrical connections. The plane of current density images and presentation of numerical results is depicted with dashed line.

$x y$-plane), mice were imaged at two orientations $90^{\circ}$ apart about $\mathrm{z}$-azis, as required by theory.

CDI images qualitatively show the effect of the two different electrode sets on the current density spatial distribution through the tumor. Figures 3a and 3c are conventional MR images, where anatomical structures of the mouse can be seen, with current density maps superimposed in the tumor area. In the $2+1$ electrode set, a small region of higher signal intensity was observed in the CDI image (Figure 3a), which corresponds to higher current density and thus to higher electric field intensity. CDI signal was decreasing towards the opposite side of the imaged plane, where the unconnected electrode was placed. When all four electrodes were connected to the voltage amplifier ( $2+2$ electrode set) current density throughout the imaging plane can be observed with two regions of higher current density (Fig. 3c) and thus higher electric field intensity. Qualitatively similar results in spatial distribution of current density were obtained by calculating current density in the observed plane of the finite element model at $50 \mathrm{~V}$ for $2+1$ electrode set (Fig. 3b) and $2+2$ electrode set (Fig. 3d). The results clearly show that spatial distribution of current density obtained by means of CDI is qualitatively similar to the calculated spatial distribution of current density in the observed plane using 3D FE model of a mouse with subcutaneous solid tumor.

In addition, the current measured during CDI was $4.7 \pm 0.3 \mathrm{~mA}$ and 

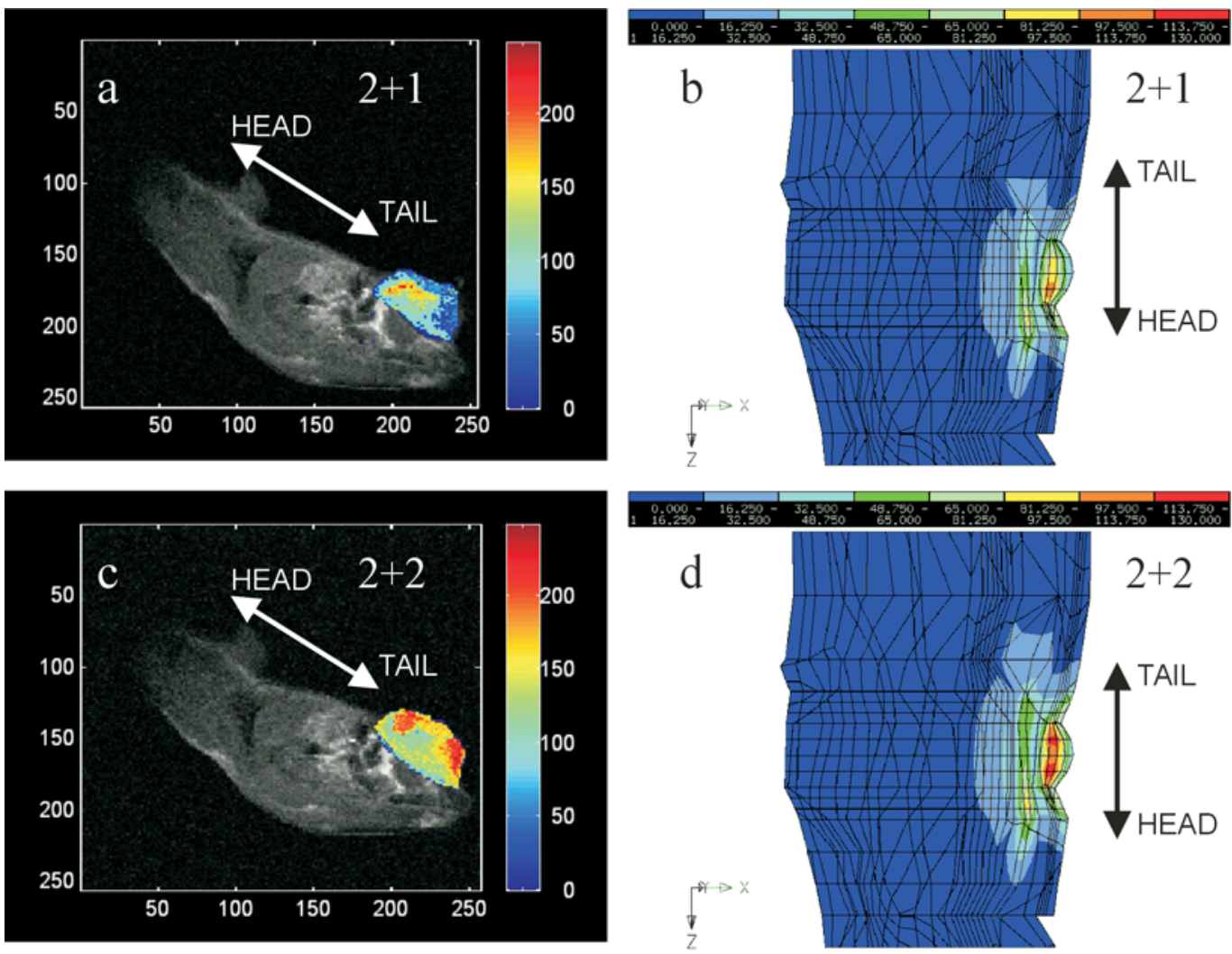

Fig. 3. Two-dimensional current density spatial distribution: (A) current density imaging (CDI) map using 2+1 electrode set (C) using 2+2 electrode set, both superimposed on a conventional MR images of a mouse with solid subcutaneous tumor; (B) calculated current densities from the finite element 3D model for $2+1$ and (D) $2+2$ electrode set. For CDI images current density is given in arbitrary units, i.e. pixel intensity ( $a$ and $c$ ). Calculated current density is given in $A / m$ (b and d).

$6.3 \pm 0.6 \mathrm{~mA}$ (mean \pm standard deviation) in $2+1$ and $2+2$ electrode set, respectively at voltage being kept constant at $50 \mathrm{~V}$. The total current in the model for $50 \mathrm{~V}$ was $4.5 \mathrm{~mA}$ and $6.1 \mathrm{~mA}$ for $2+1$ and $2+2$ electrode set, respectively.

The above validation atempts demonstrate that numerical model is reliable and can be very useful in further search for electrodes which would make electrochemotherapy and in vivo electroporation in general even more efficient. 
Table 2

Mean Values of the Magnitude of Electric Field Intensity Compared to the Antitumor Effectiveness of Electrochemotherapy with 2+1 and 2+2 Point Electrode Set

\begin{tabular}{lcc}
\hline Electrochemotherapy & $E_{\text {mean }}$ Inside Tumor $(\mathrm{V} / \mathrm{cm})$ & Growth Delay (days) \\
\hline $2+1$ electrode set & 202 & $7.7 \pm 1.3$ \\
$2+2$ electrode set & 251 & $14.0 \pm 2.0$ \\
\hline
\end{tabular}

\subsection{Effect of Electric Field Distribution on Electroporation: Comparison of Numerical and Experimental Results}

In order to further validate our numerical model we performed electrochemotherapy with $2+1$ and $2+2$ point electrodes on solid subcutaneous fibrosarcoma SA-1 tumors in A/J mice. Eight square pulses of $1300 \mathrm{~V}, 100 \mu \mathrm{s}$, repetition frequency $1 \mathrm{~Hz}$ were delivered 3 minutes after intravenous injection of $5 \mathrm{mg} / \mathrm{kg}$ bleomycin. Control experimental groups comprised bleomycin alone, electric pulses alone and not treated tumors. As excpected from numerical results and current density images, electrochemotherapy with $2+2$ electrode set was more efficient than $2+1$ electrode set (Table 2).

Distribution of scalar electric potential was also calculated for cranial/ caudal and dorsal/ventral plate electrodes configuration. Distribution of electric field intensity was then calculated from the values of the scalar electric potential in the grid points of the model. For the electrode configuration $4+4$, where for the last 4 pulses the electrodes were oriented perpendicularly with respect to the position of the electrodes for the first 4 pulses, electric field distribution was determined as a combination of the results for the cranial/ caudal and dorsal/ventral electrode configurations. Since electroporation is a threshold phenomenon, it can be assumed that in the $4+4$ electrode configuration the effective magnitude of electric field intensity in each finite element of the model is the highest of the magnitudes for cranial/caudal and dorsal/ventral electrode configurations in that particular element. Based on this assumption we determined the values of the electric field intensity for all 7089 finite elements of the model.

The results were observed in the cross section plane through the middle of the tumor alongside it (see Fig. 1), since we were most interested in the electrical phenomena inside tumor tissue. In Fig. 4 the results for the all three electrode configurations are shown. In both cranial/caudal and dorsal/ventral electrode configurations, the maximum magnitude of electric field intensity 

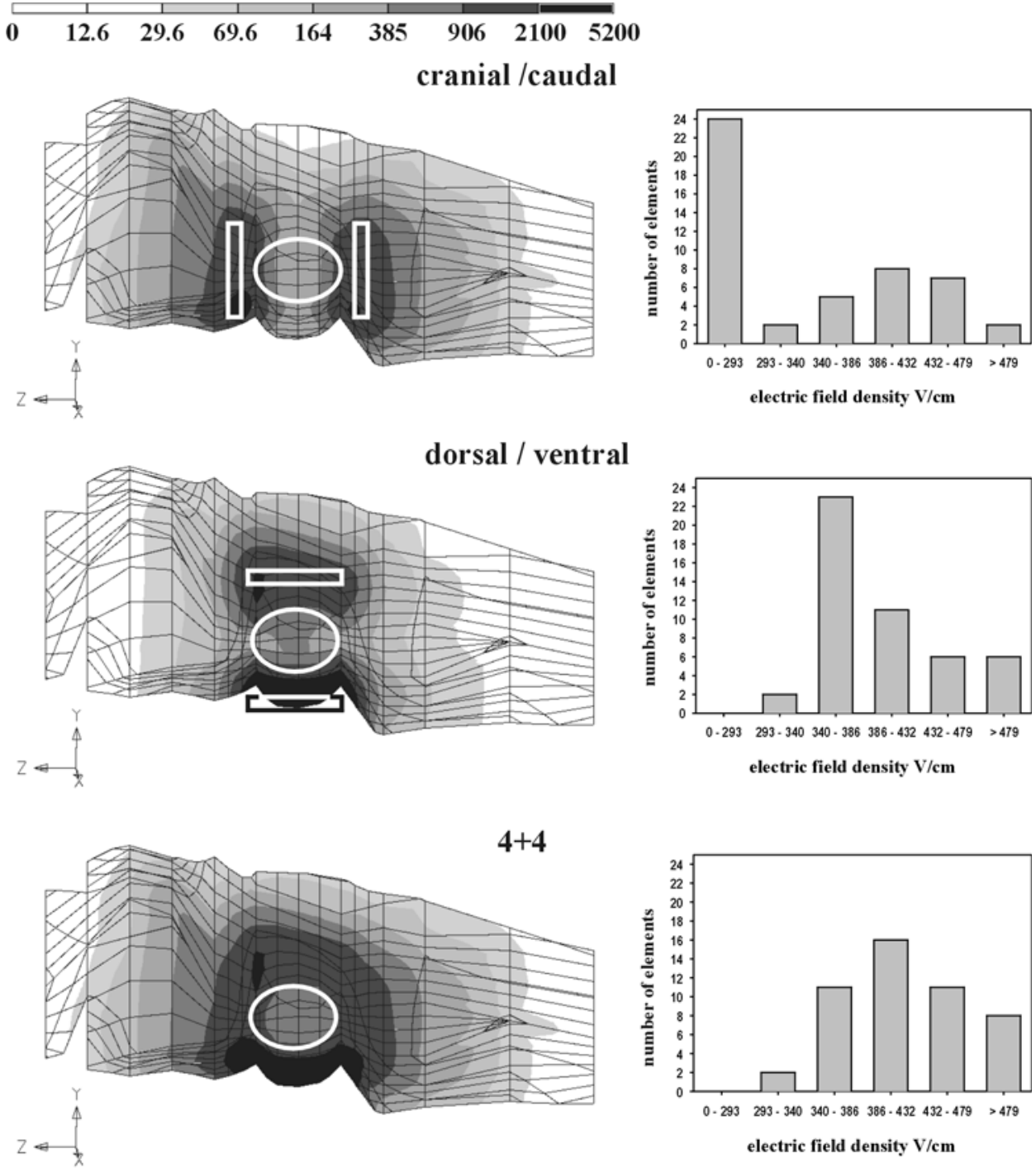

Fig. 4. Electric field distribution (from the top) for the cranial/caudal, dorsal/ ventral and 4+4 electrode configurations in the observed cross section (left) and histograms of electric field intensity in total 48 elements representing the tumor in 3D finite element model (right). Projection of the electrodes to the observed cross section and tumor location is marked in cranial/caudal and dorsal/ventral electrode configurations. 


\section{Table 3}

Mean Values of the Magnitude of Electric Field Intensity Compared to the Antitumor Effectiveness of Electrochemotherapy With Electrodes in Different Orientations and Changing of the Electrodes, i.e., 4+4 electrode configuration

\begin{tabular}{lcc}
\hline Electrochemotherapy & $E_{\text {mean }}$ Inside Tumor $(\mathrm{V} / \mathrm{cm})$ & Growth Delay (days) \\
\hline Cranial/caudal & 327 & $17.10 \pm 1.60$ \\
Dorsal/ventral & 406 & $21.35 \pm 1.94$ \\
$4+4$ & 425 & $26.17 \pm 2.32$ \\
\hline
\end{tabular}

inside the tumor is obtained on the opposite sides in proximity of the electrodes. It is much lower in the middle of the tumor and it falls even more towards edges of the tumor in the direction away from the line connecting electrodes. This pattern is in good agreement with the experimental results. Namely, in the group where electrode orientation was not changed many of the tumors regrew after successful treatment in the margins where there was no contact with the electrodes (10) (see Chapter 14 in ref. 3).

The distribution of the electric field was more precisely studied for 48 elements representing subcutaneous tumor since we were most interested in the electrical phenomena inside tumor tissue. The values of electric field intensity are presented in Fig. 4 in the form of histograms for each of the eelectrode orientations. The summary of the data together with experimental results, i.e. growth delays are listed in Table 3. Good agreement between the level of antitumor effectiveness and the mean value of electric field intensity for each particular electrochemotherapy treatment regime can be noted.

\section{Summary and Conclusions}

Mathematical modeling for in vivo electroporation has proven to be simple and efficient tool for the analysis of electrical phenomena inside biological tissue. It is very useful for the explanation of experimental results and analysis of different electroporation regimes. It was also demonstrated, that better coverage of tumors with sufficiently high electric field is necessary for improved effectiveness of electrochemotherapy and so this approach can be very useful in further search for electrodes which would make electrochemotherapy and in vivo electroporation in general more efficient. The objective of such studies would be to optimize electrode configuration in order to obtain electric fields over threshold value in the whole selected tissue, for example, tumor, and minimize it in the surrounding and deeper tissues.

Validated mathematical models make possible analysis and optimization of the shape, position and dimensions of the electrodes used in electrochemo- 
therapy for its better antitumor effectiveness and reduction of the required quantity of chemotherapeutic drug for the successful therapy that will reduce its undesired side effects on the healthy tissue. Furthermore they can help to minimize other possible side effects (pain, contraction of the surrounding muscles, influence of the induced currents on the deeper lying tissues and organs). Mathematical modeling can also provide information about electric currents in deeper lying organs, e.g. heart, and will be very useful in studies of safety measures that need to be taken in clinical use. In addition, the results obtained are also relevant for in vivo gene transfection by means of electroporation.

Mathematical modeling can be very useful in the transfer of the knowledge gained in experimental work into clinical practice. With its contribution to the development of electrochemotherapy and electro gene transfection it can bring benefits to individual patients as well as to the society. Electrochemotherapy is an attractive approach to the treatment of cancer not only because it seems to be effective in local tumor control, but also because it is an approach that can help to circumvent the side effects of chemotherapy. This would reduce the costs of cancer treatment induced by the care of the side effects, which are frequently responsible for prolonged stays in the hospitals and large consumption of drugs other than the cytotoxic drugs. With its possibilities in the optimization of the electrochemotherapeutic regimes and local in vivo gene transfection, mathematical modeling can substantially contribute to this aims. The long term perspective of mathematical modeling is to contribute to understanding and wider clinical applicability of electrochemotherapy in treatment of cancer and of electro gene transfection as a future treatment for various diseases.

\section{Acknowledgments}

This work was performed in close collaboration with Gregor Serša and Maja Čemažar from Institute of Oncology, Ljubljana, Franci Demšar, Katarina Beravs, and Igor Serša from Institute Jošef Stefan, Ljubljana. The authors would also like to thank to Alenka Maček-Lebar, Tadej Kotnik, Vojko Valenčič, Tomaž Jarm, Jani Pušenjak, Rudi Kragelj, and Lojze Vodovnik which contributed their skills and expertise in various phases of this work. The research work has been supported under various grants from The Ministry of Science and Technology of The Republic of Slovenia and PROTEUS programme of scientific, technological and cultural cooperetion between the Republic of France and the Republic of Slovenia. 


\section{References}

1. Kotnik, T., Bobanović, F., and Miklavčič, D. (1997) Sensitivity of transmembrane voltage induced by applied electric fields: A theoretical analysis. Bioelectrochem. Bioenerg. 43, 285-291.

2. Orlowski, S. and Mir, L. M. (1993) Cell electroporation: A new tool for biochemical and pharmacological studies. Biochim. Biophys. Acta 1154, 51-63.

3. Čemažar, M., Jarm, T., Miklavčič, D., Maček-Lebar, A., Ihan, A., Kopitar, N. A., and Serša, G. (1998) Effect of electric field intensity on electropermeabilization and electrosensitivity of various tumor-cell lines in vitro. Electr. Magnetobiol. 17, 261-270.

4. Belehradek, J., Orlowski, S., Ramirez, L. H., Pron, G., Poddevin, B., and Mir, L. M. (1994) Electroporation of cells in tissues assesed by the qualitative and quantitative electroloading of bleomycin. Biochim. Biophys. Acta 1190, 155-163.

5. Weaver, J. C. (1993) Electroporation: A general phenomenon for manipulating cells and tissues. J. Cell. Biochem. 51, 426-435.

6. Orlowski, S., Belehradek, J. Jr., Paoletti, C., and Mir, L. M. (1988) Transient electroporation of cells in culture. Biochem. Pharmacol. 37, 4727-4733.

7. Susil, R., Šemrov, D,. and Miklavčič, D. (1998) Electric field induced transmembrane potential depends on cell density and organisation. Electr. Magnetobiol. 17, 391-399.

8. Puc, M., Reberšek, S., and Miklavčič, D. (1997) Requirements for a clinical electrochemotherapy device: Electroporator. Radiol. Oncol. 31, 368-373.

9. Van De Graaff, K. M. and Rhees, R. W. (1987) Schaum's Outline of Theory and Problems of Human Anatomy and Physiology. McGraw-Hill, New York, pp. 39-53.

10. Serša, G., Čemažar, M., Šemrov, D., and Miklavčič, D. (1996) Changing electrode orientation improves the efficacy of electrochemotherapy of solid tumors in mice. Bioelectrochem. Bioenerg. 39, 61-66.

11. Gilbert, R. A., Jaroszeski, M. J., and Heller, R. (1997) Novel electrode designs for electrochemotherapy. Biochim. Biophys. Acta 1334, 9-14.

12. Barr, R. C., Pilkington, T. C., Boineau, J. P., and Spach, M. S. (1966) Determining surface potentials from current dipoles, with application to electrocardiography. IEEE Trans. Biomed. Eng. 13, 88-92.

13. Neonen, J. T. (1994) Solving the inverse problem in magnetocardiography. IEEE Eng. Med. Biol. 13, 487-496.

14. MacLeod, R. S. and Brooks, D. H. (1998) Recent progress in inverse problems in electrocardiology. IEEE Eng. Med. Biol. 17, 73-83.

15. Panescu, D., Webster, J. G., Tompkins, W. J., and Stratbucker, R. A. (1995) Optimisation of transcutaneous cardiac pacing by three-dimensional finite element modeling of the human thorax. Med. Biol. Eng. Comp. 33, 769-775.

16. Hutchinson, S. A., Kwong, T. Ng., Shadid, J. N., and Nadeem, A. (1997) Electri- 
cal defibrillation optimization: An automated, iterative parallel finite-element approach. IEEE Trans. Biomed. Eng. 44, 278-289.

17. Haueisen, J., Ramon, C., Eiselt, M., Brauer, H., and Nowak, H. (1997) Influence of tissue resistivities on neuromagnetic fields and electric potentials studied with a finite element model of the head. IEEE Trans. Biomed. Eng. 44, 727-735.

18. Awada, K. A., Jackson, D. R., Williams, J. T., Wilton, D. R., Baumann, S. B., and Papanicolau, A. C. (1997) Computational aspects of finite element modeling in EEG source localization. IEEE Trans. Biomed. Eng. 44, 736-752.

19. Eaton, H. (1992) Electric field induced in a spherical volume conductor from arbitrary coils: application to magnetic stimulation and MEG. Med. Biol. Eng. Comp. 30, 433-440.

20. Ferdjallah, M., Bostock, F. X., and Barr, R. E. (1996) Potential and current density distributions of cranial electrotherapy stimulation (CES) in a four-concentricspheres model. IEEE Trans. Biomed. Eng. 43, 939-943.

21. Gentili, G. B., Leoncini, M., Trembly, B. S., and Schweizer, S. E. (1997) FDTD electromagnetic and thermal analysis of interstitial hyperthermic applicators. IEEE Trans. Biomed. Eng. 42, 973-980.

22. Tsuda, N., Kuroda, K., and Suzuki, Y. (1996) An inverse method to optimize heating conditions in RF-capacitive hyperthermia. IEEE Trans. Biomed. Eng. 43, 1029-1037.

23. Gandhi, O. P. and Deford, J. F. (1988) Calculation of EM power deposition for operator exposure to RF induction heaters. IEEE Trans. Biomed. Eng. 30, 63-68.

24. Kuster, N (1993) Multiple multipole method for simulating EM problems involving biological bodies. IEEE Trans. Biomed. Eng. 40, 611-620.

25. Plonsey, R. and Heppner, D. (1967) Considerations of quasi-stationarity in electrophysiological systems. Bull. Math. Biophys. 29, 657-664.

26. Šemrov, D., Karba, R., and Valenčič, V. (1997) DC electrical stimulation for chronic wound healing enhancement. Part 2. Parameter determination by numerical modeling. Bioelectrochem. Bioenerg. 43, 271-277.

27. Miklavčič, D., Šemrov, D., Valenčič, V., Serša, G., and Vodovnik, L. (1997) Tumor treatment by direct electric current: Computation of electric current and power density distribution. Electr. Magnetobiol. 16, 119-128.

28. Geddes, L. A. and Baker, L. E. (1967) The specific resistence of biological material: A compendium of data for the biomedical engineer and physiologist. Med. Biol. Eng. 5, 271-293.

29. Foster, K. R. (1995) Dielectric properties of tissues. The Biomedical Engineering Handbook (Bronzino, J. D., ed.). CRC Press and IEEE Press, Boca Raton, FL and New York, pp. 1385-1394.

30. Brauer, J. R. (1988) What Every Engineer Should Know About Finite Element Analysis. Marcel Dekker, New York.

31. Zienkiewicz, O. C. (1977) The Finite Element Method. McGraw-Hill, New York.

32. Šemrov, D. and Miklavčič, D. (1998) Calculation of the electrical parameters in electrochemotherapy of solid tumors in mice. Comp. Biol. Med. 28, 439-448. 
33. Miklavčič, D., Beravs, K., Šemrov, D., Čemažar, M., Demšar, F., and Serša, G. (1998) The importance of electric field distribution for effective in vivo electroporation. Biophys. J. 74, 2152-2158.

34. Joy, M., Scott, G., and Henkelman, M. (1989) In vivo detection of applied electric currents by magnetic resonance imaging. Magn. Reson. Imaging 7, 89-94.

35. Serša, I., Jarh, O., and Demšar, F. (1994) Magnetic resonance microscopy of electric currents. J. Magn. Reson. Ser. A 111, 93-99. 